\title{
A Comprehensive Analysis of Different SRAM Cell Topologies in 7-nm FinFET Technology
}

\section{Erfan Abbasian}

Babol Noshirvani University of Technology

Shilpi Birla ( $\square$ shilpibirla@gmail.com )

Manipal University Jaipur https://orcid.org/0000-0002-4239-4912

\section{Morteza Gholipour}

Babol Noshirvani University of Technology

\section{Research Article}

Keywords: SRAM, Stability, CMOS, Power, FinFET.

Posted Date: August 19th, 2021

DOI: https://doi.org/10.21203/rs.3.rs-801530/v1

License: (1) This work is licensed under a Creative Commons Attribution 4.0 International License. Read Full License 


\section{Abstract}

This article aims to provide a wide comparative analysis of different SRAM cells in 7nm FinFET Technology. Due to various limitations of the CMOS device in nanotechnology, FinFET is one of the trending choices for memory designers which can improve the stability and minimize the Short Channel Effects of the CMOS. Here, performance metrics of these SRAM cells like the stability, access time, power have been measured at a supply voltage range of $0.2 \mathrm{~V}$ to $0.5 \mathrm{~V}$. The layout of the existing cells has been designed and compared in which the ST12T consumes the maximum area. The simulated results inferred that the ST11T SRAM cell offers the highest RSNM and the minimum dynamic and leakage power amongst all the SRAM cells. Moreover, ST12T SRAM cell has the highest WSNM in comparison to other SRAM cells. An electrical quality metric has been utilized, which displays the superiority of the ST12T SRAM cell amongst all the considered SRAM cells in this article.

\section{Introduction}

Shrinking of the device size leads to a dramatic rise in the demand for handheld devices. These hand devices require random access memory (RAM) as one of the major components. The portable batteryoperated systems have the foremost need the efficient designs, which should be stable, power, and area efficient. Static RAM (SRAM) is one of the best choices for them and CMOS is considered as the best choice for SRAM but due to its aggressive scaling, the device performance has degraded due to short channel effects (SCE).

Scaling imposed physical limitations on CMOS: As scaling is done, it is important to maintain the electric field, the thickness of the gate oxide needs to be reduced which gives rise to the leakage current exponentially as the gate oxide thickness is reduced. The rising tunneling current may affect devices adversely. The smaller channel length is supposed to have faster switching operations but at the same time it has adverse effects: it rises the OFF-current ( $\left.\mathrm{I}_{\text {off }}\right)$ which results in punch through and drain induced barrier lowering (DIBL) (Haron, 2008). The other factors which are affected by the scaling are the supply voltage and the threshold voltage, though the scaling of the supply voltage leads to a drastic reduction in dynamic power reduction, it leads to degradation of the noise margin. Due to supply voltage scaling the threshold voltage requires to be scaled which results in the operation of the transistor in weak inversion, giving rises to subthreshold leakage current. To minimize short channel effects, an increase in channel doping is required, this raises other effects like the band to band tunneling (BTBT) and slow carrier mobility (Frank, 2001). For reduction in power consumption, scaling or reducing the supply voltage is one of the utmost accepted solutions, but it leads to degraded stability in terms of read margin which results in read failure. Also, scaling of supply voltage leads to threshold voltage variations which are further responsible for increasing the leakage current improve the performance, one of them is to move from a single port to multiport SRAM cells. Since the basic 6T SRAM Cell was the single port cell that suffers from read failure due to scaling of the CMOS. Suggestions to improve the performance were given in the sizing of the transistors ratios which also failed due to aggressive scaling and low supply voltage. 
In due course of time researchers started thinking for an alternative of a CMOS device that should be nano in size and can combat the process variations. Researchers classified the nanodevices in three categories: (1) "Electric Dependent Nanodevices: These nanodevices may be having either ballistic, tunneling, or electrostatic mechanism. In ballistic transport, the electrons move with no resistivity in the material. As a result of a quantum-mechanical process, electrons flow through a potential energy barrier in tunneling. In electrostatic, the electric field plays an important role as the electrons move in the electric field only. Some of them area carbon nanotube field-effect transistors (CNTEFTs), fin shaped field effect transistors (FinFETs), nanowire field effect transistors (NWFETs), tunnel field effect transistors (TFETs) (Goser, 2004) (Zhirnov, Emerging research logic devices, 2005). (2) Magnetic Dependent Nanodevices: The mode of "Magnetostatic" and "spin transport" comes under this category. magnetic quantum-dot cellular automata (MQCA) and spin field-effect transistors (spinFETs) are few examples (Zhirnov, Emerging research logic devices, 2005) (Goser, 2004). (3) "Mechanical-Dependent Nanodevices" used conductive polymers. The polymer structure varies when they are activated by the inputs (Goser, 2004), these sorts of devices are being used in field programmable gate arrays (FPGAs) and molecular memory (Cotofana).

All the devices mentioned above possess some advantages over the CMOS. SRAM is more sensitive to the process variations in the physical structure, variation of the threshold voltage because of the dopant fluctuation. So, designing an SRAM that is least affected by these variations is a big challenge in CMOS designs. One of the most favored alternatives found for CMOS was FinFET as it consists of all the features of the CMOS along with the potential to combat the SCEs. The FinFET is preferred as it exhibits excellent gate control, has low switching times, and high current density compared to the CMOS devices. CMOS is a planar device where the gate control is only in one direction while in FinFET the gate is wrapped all around the gate which provides excellent gate control in all directions. The body of the FinFET is very thin due to which the gate capacitance lies close to the channel which helps in controlling the leakage. The device drive current of the FinFET is increased by forming parallel multiple fins connected, so the channel length is not the only parameter for the driving current. Besides, FinFET has the potential to increase carrier mobility using strain technology. It also has a low $\mathrm{I}_{\text {off }}$ current which further helps in reducing the leakage.

Therefore, study and evaluation of FinFET performance, as a prominent replacement for CMOS, in digital circuits including SRAM cells is necessary. This paper comprehensively compares different SRAM cell topologies that include the conventional 6T, conventional 8T, Schmitt-Trigger-based 10T (ST2), P-P-Nbased cell core 10T (PPN10T), single-ended feedback-cutting 11T (FC11T), Schmitt-Trigger-based 11T (ST11T), and Schmitt-Trigger-based 12T (ST12T) in terms of stabilities, delays, dynamic/leakage power consumption, area, and quality metric. The considered SRAM cells have been redesigned using 7-nm FinFET technology. The attained characteristics of different SRAM cell topologies are summarized as:

1. The ST11T and ST12T have the similar highest HSNM as they use two cross-coupled ST-based inverters pair. 
2. The ST11T SRAM cell employing the read decoupling technique and strong cell core offers the highest RSNM.

3. The highest WSNM is related to the ST12T SRAM cell because of the differential writing structure along with utilizing the power-gating write-assist technique.

4. Two conventional 6T and 8T SRAM cells show the same lowest write delay due to their simple differential writing structure formed by only one access NMOS pass-gate transistor.

5. The conventional 6T SRAM cell has the lowest read delay attributed to its differential reading structure including a strong pull-down transistor.

6. The ST11T SRAM cell dissipates the least dynamic power as it has single-ended structure and relatively low speed.

7. The least leakage power dissipation is related to the ST12T SRAM cell due to the presence of a stacked transistor in its cell core.

8. The conventional 6T SRAM cell occupies the minimum area among all the considered SRAM cells.

9. The ST12T SRAM cell has the highest electrical quality metric, as a complete metric to estimate the total performance of an SRAM cell.

The rest of this paper is as follows. Section 2 reviews in detail about the different SRAM cell topologies considered for comparison in this study, simulation results and analysis are given in section 3 . The conclusion of this article is discussed in section 4 .

\section{Literature Review}

The conventional 6T SRAM cell shown in Fig. 1(a)) has a simple and compact structure consisting of two cross-coupled inverters pair to form its cell core and two n-type MOS (NMOS) access pass gate transistors to connect two-bit lines (BL and BLB) to the cell. The conventional 6T SRAM cell uses the same path for performing its read and writes operation, therefore, suffering extremely from conflicting read/write necessities. The conventional 6T SRAM cell shows poor read static noise margin (RSNM)/write static noise margin (WSNM), as a measure of read stability/writability, in severe low-supply voltage (Golipour, 2021). For the common 6T SRAM cell, RSNM and WSNM are two conflicting design metrics. Therefore, it is intensely difficult to utilize the 6T SRAM cell in severe low-supply voltage, especially below the transistor's threshold voltage.

In (L. Chang, 2005), basic 8T SRAM cell (see Fig. 1(b)) is proposed to remove the read disturbance in the 6T SRAM cell. The 8T SRAM cell uses two NMOS access transistors extra, one read word line, and one read bit line compared to the conventional 6T SRAM cell to form the isolated read path. During the read operation data storing nodes are fully decoupled from the read bit line to enhance RSNM. However, this cell suffers from the leakage introduced by the isolated read path, which is further significant with technology scaling. A drastic improvement has been observed with a slight area overhead of $30 \%$. 
In (Kulkarni, 2011), to solve the read stability issues, the basic principle of the Schmitt-Trigger (ST) has been used. In this work, the authors proposed two types of ST-based SRAM cells for improving cell stability. The feedback mechanism is used in the ST1 SRAM cell, but the limitation in the ST1 bit cell, is that the feedback is useful till voltage on the storing nodes is maintained, once the voltages start discharging or charging this feedback mechanism is no more effective. the feedback mechanism is useful if the storage node voltages are maintained. To remove this limitation, the ST2 SRAM cell is projected, displayed in Fig. 1(c)). A separate control signal is used for the improvement in the feedback mechanism. This differential sensing mechanism has been used with two separate word lines (WL and $W W L$ ) along with two different bit lines (BL and BLB). This bit cell is effective for low voltage operations, but it has area overhead while the other read/write assist techniques have a lower area overhead.

A PMOS-PMOS-NMOS-based cell core 10T (PPN10T) SRAM cell is projected in (Sanvale, 2019)and shown in Fig. 1(d). This cell employs differential writing and single-ended reading structures for its write and read operation, respectively. This cell can improve RSNM/WSNM by using an isolated read path/differential writing path. The leakage power dissipation is reduced by this cell due to the presence of a stacked transistor in its cell core. However, it consumes high dynamic write power attributed to its dualended write bit lines.

In (Ensan, 2018), a feedback-cutting 11T FinFET SRAM cell (namely FC11T) is proposed and shown in Fig. 1(e), which is a single-ended robust cell. The FC11T SRAM cell has single bit line to perform read and write operations, therefore, resulting in dynamic power reduction. This cell can improve both RSNM and WSNM by employing isolated read path and feedback-cutting write-assist technique, respectively. However, these improvements are at the cost of lower reading and writing speed. This cell is found robust for the near subthreshold operation.

Another ST-based SRAM cell employing 11 transistors (ST11T) is proposed in (Ahmad, 2016) (see Fig. $1(f))$ to improve both RSNM and WSNM and to reduce power consumption. Improvements in the RSNM, WSNM, and power consumption are because of the read decoupling technique, virtual grounded writeassist technique, and single-ended structure, respectively. However, this cell employs the same isolated read path as the conventional 8T SRAM cell, which shows higher leakage in this path. The layout has been designed at $45 \mathrm{~nm}$ technology rules showing an increase of $2 \mathrm{X}$ times area in comparison to $6 \mathrm{~T}$ SRAM cell.

An ST-based 12T (ST12T) SRAM cell is discussed in (Tomar, 2020) (see Fig. 1(g)), which employs singleended reading and differential writing structures. This cell still suffers from read disturbance but shows high WSNM , since it uses power gating write-assist technique. This cell can improve the dynamic read power consumption with the aid of a single-ended reading operation. However, this cell suffers from the area overhead.

\section{Simulation Results And Discussions}


Different SRAM cell topologies including the conventional 6T, conventional 8T (L. Chang, 2005) , SchmittTrigger-based 10T (ST2) (Kulkarni, 2011), P-P-N-based cell core 10T (PPN10T) (Sanvale, 2019), singleended feedback-cutting 11T (FC11T) (Ensan, 2018), Schmitt-Trigger-based 11T (ST11T) (Ahmad, 2016), and Schmitt-Trigger-based 12T (ST12T) (Tomar, 2020), as shown in Fig. 1, are investigated in this section. Table 1 compares the cell features of the SRAM cells. These SRAM cells are redesigned using FinFET devices and comprehensively compared in terms of major design metrics like stability factors and delay during the working modes, dynamic power, leakage power, and quality metric. For this reason, various HSPICE simulations at 7-nm predictive technology model multi-gate (PTM-MG) technology (P. T. M. (PTM)) has been done at $L E V E L=72,25^{\circ} \mathrm{C}$ temperature, and a certain range of supply voltage of $V_{D D}$ from $0.2 \mathrm{~V}$ to $0.5 \mathrm{~V}$ by a linear variation of $0.1 \mathrm{~V}$. The effective width (W) of a FinFET is given in Eq. (1) as follows (Mahmoodi, 2020):

$$
\mathrm{W}=\mathrm{N}\left(2 \times \mathrm{H}_{\text {fin }}+\mathrm{T}_{\text {fin }}\right)
$$

in which $\mathrm{H}_{\mathrm{fin}}$ is the fin height and $\mathrm{T}_{\text {fin }}$ is the fin thickness.

Transistor sizing plays a vital role in an SRAM cell design, which highly affects the RSNM and WSNM of that cell. This issue becomes significant in the 6T SRAM cell because of conflicting read/write requirements (Golipour, 2021). Therefore, in all the considered SRAM cells, an $N=2$ and an $N=1$ have been chosen for pull-down transistors and other remaining transistors, respectively (Ansari, 2015). A comparative analysis of different performance metrics is discussed in the following subsections.

Table 1. Feature comparison of various studied SRAM cells. 


\begin{tabular}{|c|c|c|c|c|c|c|c|}
\hline Cell Feature & $6 T$ & $8 T$ & ST2 & PPN10T & FC11T & ST11T & ST12T \\
\hline $\begin{array}{l}\text { Transistor } \\
\text { Count }\end{array}$ & 6 & 8 & 10 & 10 & 11 & 11 & 12 \\
\hline Read/Write & $\operatorname{Diff}^{\mathrm{a}} / \mathrm{Diff}$ & SE $E^{b} /$ iff & Diff/Diff & Diff/Diff & SE/SE & SE/SE & SE/Diff \\
\hline \multirow[t]{2}{*}{ Bitlines } & $(2)$ & (3) & (2) & (3) & (1) & (2) & (2) \\
\hline & BL-BLB & $\begin{array}{l}\text { BL-BLB- } \\
\text { RBL }\end{array}$ & BL-BLB & $\begin{array}{l}\text { WBL- } \\
\text { WBLB- } \\
\text { RBL }\end{array}$ & $\mathrm{BL}$ & BL-RBL & BL-BLB \\
\hline \multirow{2}{*}{$\begin{array}{l}\text { Control } \\
\text { signals }\end{array}$} & (1) & $(2)$ & (2) & (2) & (3) & (3) & (3) \\
\hline & WL & WL-RWL & $\begin{array}{l}\text { WL- } \\
\text { WWL }\end{array}$ & WL-RWL & $\begin{array}{l}\text { WL- } \\
\text { WWLA- } \\
\text { WWLB }\end{array}$ & $\begin{array}{l}\text { WL-RWL- } \\
V_{\text {GND }}\end{array}$ & $\begin{array}{l}\text { WL- } \\
\text { WLB- } \\
\text { RWL }\end{array}$ \\
\hline $\begin{array}{l}\text { Read- } \\
\text { disturb-free }\end{array}$ & No & Yes & No & Yes & Yes & Yes & No \\
\hline $\begin{array}{l}\text { Half-select- } \\
\text { free }\end{array}$ & No & No & No & No & No & Yes & Yes \\
\hline
\end{tabular}

\subsection{Stability Analysis}

The stability is normally estimated as the static noise margin (SNM). The noise margin is characterized by plotting the overlapped voltage transfer characteristics (VTCs) (known as butterfly curves) for the cross-coupled inverters that make the latch of memory cells (Sanvale, 2019). The length of the biggest square which can be inscribed inside the smaller eye of the butterfly curve provides the noise margin (Gholipour E. A., 2020). Table 2 gives the SNM of the various SRAM cells in the hold, read, and write modes at $\mathrm{V}_{\mathrm{DD}}=0.2 \mathrm{~V}$.

Table 2. A static noise margin of the various SRAM cells during the hold, read, and write operations at $\mathrm{V}_{\mathrm{DD}}=0.2 \mathrm{~V}$.

\begin{tabular}{llllllll} 
Static noise margin & $\mathbf{6 T}$ & $\mathbf{8 T}$ & $\mathbf{S T 2}$ & PPN10T & FC11T & ST11T & ST12T \\
\hline HSNM (mV) & 67.1 & 67.1 & 73.5 & 62.4 & 67.1 & 79.2 & 79.2 \\
\hline RSNM (mV) & 32.8 & 67.1 & 46.3 & 62.4 & 67.1 & 79.2 & 44.1 \\
\hline WSNM (mV) & 73.4 & 46.9 & 89 & 67 & 84.5 & 74 & 112.3
\end{tabular}

\subsubsection{Hold Stability}

Hold stability of an SRAM cell is gauged by the hold static noise margin (HSNM) and defined as the maximum DC noise voltage that the SRAM cell can stand without altering the data in the hold mode (He, 
2019). HSNM is the side length of the maximum square which can fit in the smaller wing of the butterfly curve during the hold mode (Gholipour A. a., 2020). Fig. 2 displays the hold butterfly curves of the considered SRAM cells in this study at $\mathrm{V}_{\mathrm{DD}}=0.2 \mathrm{~V}$. Moreover, Fig. 3 exhibits the HSNM variations of all the tested SRAM cells against linear variations in the $V_{D D}$. It is observed from Fig. 3 that the HSNM value increases with the $\mathrm{V}_{D D}$ increment. SRAM cells including the conventional $6 T$, conventional $8 T$, and FC11T employ conventional cell core formed by cross-coupled normal inverters pair, and therefore show the same and lower HSNM compared to ST2, ST11T, and ST12T SRAM cells. Although, the PPN10 SRAM cell also uses a conventional cell core, however, it offers the lowest HSNM among all the studied SRAM cells as it has stacked pull-up structure. The ST2 SRAM cell employing modified Schmitt-Trigger inverter-based cell core uses stacked pull-down structure and thus, it has the second-best HSNM. Due to the use of a strong cell core consisting of two Schmitt-Trigger inverters, the ST11T and ST12T SRAM cells has equal and highest HSNM compared to all the SRAM cells at all supply voltages.

\subsubsection{Read Stability}

The read stability of an SRAM cell is estimated by the read static noise margin (RSNM). The maximum DC noise voltage which an SRAM cell can sustain without changing the data during the read mode is defined as Read Stability (He, 2019). The RSNM is considered as the length of a side of the largest square which is embedded inside the smaller eye of the butterfly curve during the read mode (Gholipour A. a., 2020). Figs. 4 and 5 show the read butterfly curves at $V_{D D}=0.2 V$ and the RSNM variations versus $V_{D D}$ variations, respectively. The conventional 6T, ST2, and ST12T SRAM cells suffer from the read disturbance issue, resulting in RSNM degradation. These SRAM cells show the lowest RSNM in comparison to other considered SRAM cells at all $V_{D D}$ values. However, the ST2 and ST12T SRAM cells show considerably higher RSNM in comparison to conventional 6T SRAM cell due to their SchmittTrigger-based inverters. Other remaining SRAM cells such as the conventional 8T, PPN10T, FC11T, and ST11T employ the read decoupling technique in which data storing nodes are fully isolated from the bitlines during the read operation, results into same values for RSNM \& HSNM. The ST11T SRAM cell displays the maximum RSNM amongst all the SRAM cells due to its strong cell core formed by crosscoupled Schmitt-Trigger-based inverters pair.

\subsubsection{Writability}

Writability of an SRAM cell is determined by the write static noise margin (WSNM), which is the capability of that SRAM cell to pull-down/up a ' $1 / /$ ' 0 ' storage node to a voltage lower/higher than the switching threshold $\left(V_{t h}\right)$ of the another inverter ' 0 ' $/{ }^{\prime} 1$ ' storing node; consequently, the state of the cell is successfully flipped (Gholipour E. A., 2020). To calculate the WSNM of an SRAM cell, first, the read VTC of that SRAM cell is combined with its write VTC, and then the length side of the smallest square that can be inserted between and lower half of these VTCs gives the WSNM of the corresponding SRAM cell. Fig. 6 shows the combined read and write VTCs of the studied SRAM cells at $V_{D D}=0.2 \mathrm{~V}$. Furthermore, the WSNM variations of the SRAM cells versus $V_{D D}$ variations given in Fig. 7. As $V_{D D}$ increases, the WSNM values also increase. SRAM cells such as FC11T, ST11T, and ST12T use feedback-cutting, power-gated, 
and floating virtual ground write-assist techniques, respectively to improve the WSNM. The conventional 8T and PPN10T SRAM cells show lower WSNM to the 6T SRAM cell due to their read VTC. In the ST2 SRAM cell, read access transistors also help to write a ' 0 ' or ' 1 ' into the cell, resulting in WSNM improvement.

\subsection{Access Time or Delay}

Access time or delay average of the low-to-high propagation delay $\left(t_{\mathrm{PLH}}\right)$ and the high-to-low propagation delay ( $\left.t_{P H L}\right)$. These are two kinds of read and write access time (Sanvale, 2019). Table 3 reports the read and write access times of the considered SRAM cells in this study.

Table 3. The speed performance of the various SRAM cells at $V_{D D}=0.2 \mathrm{~V}$.

\begin{tabular}{llllllll} 
Speed performance & $\mathbf{6 T}$ & $\mathbf{8 T}$ & $\mathbf{S T 2}$ & PPN10T & FC11T & ST11T & ST12T \\
\hline Read Delay (ns) & 2.75 & 3.64 & 5.63 & 3.64 & 4.91 & 3.64 & 4.21 \\
\hline Write Delay (ns) & 0.30 & 0.30 & 0.38 & 0.39 & 2.38 & 1.67 & 0.74
\end{tabular}

\subsubsection{Read Access Time or Read Delay}

The reading speed of an SRAM cell is gauged by the read delay or access time $\left(T_{R A}\right)$. It is defined as the time between the word line activation when the bit line voltage is discharged by $50 \mathrm{mV}$ from the original high value (Golipour, 2021) (S. Ahmad, 2017). Fig. 8 sows the comparisons of the $T_{R A}$ of the various SRAM cell at different $V_{D D}$ values. From this figure, the 6T SRAM cell demonstrates the lowest $T_{R A}$ due to its reading path, which includes the strong pull-down transistor with an $\mathrm{N}=2$. Various SRAM cell like the conventional 8T, PPN10T, and ST11T employ the same isolated read path made by two access transistors. Therefore, these SRAM cells show higher $\mathrm{T}_{\mathrm{RA}}$ in comparison to 6T SRAM cell. In the ST12T SRAM cell, the read path is created by three series-connected access transistors; consequently, this SRAM cell offers higher $T_{R A}$ as compared to the above-mentioned SRAM cells. Because of higher overall bit lines capacitance as a direct consequence of the connection of several access transistors to the same bit lines, the ST2 SRAM cell shows the highest $T_{R A}$ among all the SRAM cells at all $V_{D D}$ values.

\subsubsection{Write Access Time or Write Delay}

The swiftness of an SRAM cell during the write operation is measured by write access time or write delay $\left(T_{W A}\right)$. The $T_{W A}$ is well-defined as the time duration between the word line activation and the time when the storage node $Q(Q B)$ reaches $10 \%(90 \%)$ of $V_{D D}$. The $T_{W A}$ for writing ' 1 ' into ' 0 ' storing node $Q$ in all the SRAM cells against $V_{D D}$ variations is shown in Fig. 9. The conventional $6 \mathrm{~T}$ and $8 \mathrm{~T}$ SRAM cells have the same simple differential writing structure formed by a single access transistor. Therefore, these SRAM cells show the same lowest TWA compared to all the SRAM cells. The FC11T and ST12T SRAM cells 
show the highest $\mathrm{T}_{W A}$ among all the compared SRAM cells due to their single-ended writing operation. However, the FC11T SRAM cell offers higher TWA than ST11T SRAM cell because in the former cell the utilized feedback-cutting write-assist technique changes the cell core to be as two cascaded inverters during the write operation in which one inverter is followed by other one, resulting in $T_{W A}$ increment. In the ST12T SRAM cell, the switching threshold $\left(V_{\text {th }}\right)$ of Schmitt-Trigger inverters in the cell core is higher than the normal one. This issue increases the TWA. The SRAM cells including ST2 and PPN10T SRAM cells show almost equally lower $\mathrm{T}_{\mathrm{WA}}$ than the conventional $6 \mathrm{~T}$ SRAM cell.

\subsection{Dynamic/Leakage Power Consumption}

Dynamic/Leakage power consumption of an SRAM cell is the power consumption of the cell when it is accessed/it is in idle mode. Table 4 reports the power consumption of numerous SRAM cells at $V_{D D}=0.2$ V.

Table 4. Power consumption of the various SRAM cells at $V_{D D}=0.2 \mathrm{~V}$.

\begin{tabular}{llllllll} 
Power consumption & $\mathbf{6 T}$ & $\mathbf{8 T}$ & $\mathbf{S T 2}$ & PPN10T & FC11T & ST11T & ST12T \\
\hline Dynamic Power $(\boldsymbol{\mu W})$ & 12.335 & 13.354 & 19.794 & 10.057 & 7.761 & 3.335 & 6.248 \\
\hline Leakage Power $(\mathrm{nW})$ & 0.730 & 0.812 & 0.565 & 0.693 & 0.236 & 0.565 & 0.151
\end{tabular}

\subsubsection{Dynamic Power Consumption}

Dynamic power $\left(\mathrm{P}_{\text {Dyn }}\right)$ is the notable portion of the overall power consumed by an SRAM cell. Since the write power is dynamic power, hence dynamic power expression is used for the calculation of write power. The write power of an SRAM cell is thus given by Eq. (3):

$$
\left.P_{D y n}=\alpha_{\text {write }} \times C_{B L} \times V_{D D}^{2}+f_{\text {write }}\right)
$$

where awrite is the switching activity factor, $f$ write is writing frequency. The maximum value for Write activity factor is considered as 1 (Roy, 2020). The Simulation results of write power is shown graphically in Fig. 10. SRAM cells such as the conventional 6T, 8T, ST2, PNN10T, and ST12T employ fully differential writing structure, and therefore their $\mathrm{P}_{\text {Dyn }}$ is higher than other SRAM cells with single-ended writing structure. The ST2 SRAM cell consumes the highest $P_{\text {Dyn }}$ among all the SRAM cells due to its higher overall bit line capacitances because of the connection of several access transistors to the same bit lines. Although, the FC11T SRAM cell uses a single-bit line for performing both read and write operations, however, its bit line needs to be discharged to the ground for every write operation, and therefore it consumes higher $P_{\text {Dyn }}$. The PPN10T and ST12T SRAM cells show lower $P_{\text {Dyn }}$ than the 
conventional 6T SRAM cell due to their lower $f$ write. In comparison to all SRAM cells, the ST11T SRAM cell offers the least $\mathrm{P}_{\mathrm{Dyn}}$ as it has single-ended nature and lower $f$ write.

\subsubsection{Leakage Power Dissipation}

The leakage power $\left(P_{\text {Leak }}\right)$ is the power strained by MOS devices from $V_{D D}$ when the cell lies in the standby mode. As an SRAM cell stays maximum of the time in hold mode, therefore the $P_{\text {Leak }}$ is an important factor to total power consumption (Golipour, 2021). Fig. 11 compares the $P_{\text {Leak }}$ of the various SRAM cells at different $V_{D D}$ values. The conventional 8T SRAM cell displays a higher $P_{\text {Leak }}$ to the conventional 6T SRAM cell due to its higher count of bit lines used. Furthermore, this SRAM cell has high leakage in its isolated read path, which becomes significant with technology scaling (S. Ahmad, 2017). The ST2 and PPN10T SRAM cells dissipate lower $P_{\text {Leak }}$ than the conventional 6T SRAM cell as stacked transistors are used in their cell core. Although, the ST11T is single-ended and uses stacked transistors, however, this memory cell employs the isolated read path as the 8T SRAM cell, therefore, suffering from isolated read path's leakage. This SRAM cell displays higher $\mathrm{P}_{\text {Leak }}$ in comparison to FC11T and ST12T SRAM cells.

\subsection{Layout Area}

The layouts of the considered SRAM cells in this study, created on the design rules for the FinFET technology given in (Salahuddin, 2013) , are demonstrated in Fig. 12. The area for each cell is described in Table 5, where $\lambda$ is the minimum feature size which is considered as $1 / 2$ of the gate length (Ansari, 2015). The conventional 6T SRAM cell displays the minimum layout area because of its simple structure with a minimum count of transistors used among all the considered SRAM cells in this article, the ST12T SRAM cell, on the other hand, consumes the highest layout area, which is attributed to its higher count of transistors utilized in this design.

Table 5. The layout dimensions of the compared SRAM cells are based on $\lambda$.

\begin{tabular}{|c|c|c|c|c|}
\hline SRAM cells & Height $(\lambda)$ & Length $(\lambda)$ & Area $\left(\lambda^{2}\right)$ & Normalized area \\
\hline 6T & 24 & 39 & 936 & $1 \times$ \\
\hline $\mathbf{8 T}$ & 24 & 48 & 1152 & $1.23 \times$ \\
\hline ST2 & 24 & 72 & 1728 & $1.84 \times$ \\
\hline PPN10T & 24 & 61 & 1464 & $1.56 \times$ \\
\hline FC11T & 34 & 55 & 1870 & $1.99 \times$ \\
\hline ST11T & 24 & 71 & 1704 & $1.82 \times$ \\
\hline ST12T & 34 & 60 & 2040 & $2.17 \times$ \\
\hline
\end{tabular}

\subsection{Electrical Quality Metric}


For comprehensive evaluation of the performance of an SRAM cell, an electrical quality metric (EQM) has been proposed in (Jiao, 2016) and expressed as Eq. (4) to assess the overall excellence of a cell.

$$
\mathrm{EQM}=\frac{\mathrm{HSNM} \times \mathrm{RSNM} \times \mathrm{WSNM}}{\mathrm{T}_{\mathrm{RA}} \times \mathrm{T}_{\mathrm{WA}} \times \mathrm{P}_{\text {Dyn }} \times \mathrm{P}_{\text {Leak }} \times \text { Area }}
$$

Where, HSNM, RSNM, and WSNM are the static noise margin during the hold, read, and write operations, respectively. $T_{R A}\left(T_{W A}\right)$ is the read (write) access time. $P_{\text {Dyn }}$ is the dynamic write power consumption, $P_{\text {Leak }}$ is the leakage power dissipation, and Area is the layout area. It is observed from Fig. 13, which shows the EQM of all cells at $V_{D D}=0.2 \mathrm{~V}$, that the ST12T/ST2 SRAM cell offers the highest/lowest EQM amid all the considered SRAM cells.

\section{Conclusion}

A thorough analysis of diverse SRAM cell topologies redesigned using 7-nm FinFET technology is presented in this article. The various performance metrics of these SRAM cells such as stabilities, delays, dynamic power, and leakage power have been measured at a certain range of supply voltage from $0.2 \mathrm{~V}$ to $0.5 \mathrm{~V}$ with a linear variation of $0.1 \mathrm{~V}$ and a room temperature of $25^{\circ} \mathrm{C}$. Based on the obtained results, the ST11T SRAM cell offers the highest RSNM and the least dynamic power among all the considered SRAM cell. Furthermore, the ST12T SRAM cell shows the highest WSNM, and the least leakage power compared to other SRAM cells. This cell, on the other hand, occupies the highest area. In terms of speed performance, the conventional 6T SRAM cell has the best read and write access times due to its simple fully differential structure. To assess the overall performance of these SRAM cells, an electrical quality metric has been utilized, which displays the superiority of the ST12T SRAM cell among all the SRAM given cells in this article.

\section{Declarations}

Funding-There is no funding received for this work

Conflicts of interest/Competing interests- There is no conflict of interest at any stage.

Availability of data and material (data transparency)-The associated data will be made available on request

Code availability (software application or custom code)- The simulation work has been carried out using HSPICE software.

Authors' contributions (optional): NA Additional declarations

Ethics approval: NA It is a simulation-based design and analysis. So, it does not produce any environmental hazards. 
Consent to participate: Yes, we are ready to participate.

Consent for publication: We are ready for publication with your journal.

Acknowledgement: We acknowledge for the support and lab facility provided by Department of ECE, School of Electronics and Electrical Engineering, MANIPAL UNIVERSITY JAIPUR, INDIA

\section{References}

1. Ahmad, S. a. (2016). Single-ended Schmitt-trigger-based robust low-power SRAM cell. IEEE Transactions on Very Large Scale Integration (VLSI) Systems, 2634-2642.

2. Ansari, M. a.-K.-K. (2015). A near-threshold 7T SRAM cell with high write and read margins and low write time for sub-20 nm FinFET technologies. Integration, 91-106.

3. applications, D. o.-p. (2020). Roy, Chandramaulashwar and Islam, Aminul. Microsystem Technologies, 3299-3310.

4. cell, A. S.-t. (2020). A Schmitt-trigger based low read power 12T SRAM cell. Analog Integrated Circuits and Signal Processing, 275-295.

5. Cotofana, S. (n.d.). Emerging Phenomena-dependent Non-CMOS Nanoelectronic Devices-What Are They? 4th IEEE International Conference on Nano/Micro Engineered and Molecular Systems, IEEE-NEMS, Shenzhen, (pp. 5-8). China: IEEE.

6. Ensan, S. S. (2018). A robust and low-power near-threshold SRAM in 10-nm FinFET technology. Analog Integrated Circuits and Signal Processing, 497-506.

7. Frank, D. J.-S. (2001). Device scaling limits of Si MOSFETs and their application dependencies. Proceedings of the IEEE, 259-288.

8. Gholipour, A. a. (2020). A variation-aware design for storage cells using Schottky-barrier-type GNRFETs. Journal of Computational Electronic, 987-1001.

9. Gholipour, E. A. (2020). Design of a Schmitt-Trigger-Based 7T SRAM Cell for Variation Resilient Low-Energy consumption and Reliable Internet of Things Applications. International Journal of Electronics and Communications, 153899.

10. Golipour, E. A. (970-989). Single-ended half-select disturb-free 11T static random access memory cell for reliable and low power applications. International Journal of Circuit Theory and Applications, 2021.

11. Goser, K. P. (2004). Nanoelectronics and nanosystems: from transistors to molecular and quantum devices. Springer Science $\backslash \&$ Business Media.

12. Haron, N. Z. (2008). Why is CMOS scaling coming to an END? 3rd International Design and Test Workshop (pp. 98-103). IEEE.

13. He, Y. a. (2019). A half-select disturb-free 11T SRAM cell with built-in write/read-assist scheme for ultralow-voltage operations. IEEE Transactions on Very Large Scale Integration (VLSI) Systems, 2344-2353. 
14. Jiao, H. a. (2016). Low power and robust memory circuits with asymmetrical ground gating. Microelectronics Journal, 109-119.

15. Kulkarni, J. P. (2011). Ultralow-voltage process-variation-tolerant Schmitt-trigger-based SRAM design. IEEE transactions on very large scale integration (VLSI) systems, 319-332.

16. L. Chang, D. M. (2005). Stable SRAM cell design for the $32 \mathrm{~nm}$ node and beyond. in Digest of Technical Papers, 2005 Symposium on VLSI Technology (pp. 128-129). IEEE.

17. Mahmoodi, E. \&. (2020). Design space exploration of low-power flip-flops in FinFET technology. Integration, 52-62.

18. P. T. M. (PTM), ". A. (n.d.).

19. S. Ahmad, M. K. (2017). Low leakage single bitline $9 \mathrm{t}$ (sb9t) static random access memory. Microelectronics Journal, 1-11.

20. Salahuddin, S. a. (2013). A novel 6T SRAM cell with asymmetrically gate underlap engineered FinFETs for enhanced read data stability and write ability. International Symposium on Quality Electronic Design (ISQED) (pp. 353-358). IEEE.

21. Sanvale, P. a. (2019). An improved read-assist energy efficient single ended PPN based 10T SRAM cell for wireless sensor network. Microelectronics Journal, 104611.

22. Zhirnov, V. V. (2005). IEEE Circuits and Devices Magazine. Emerging research logic devices, 3746.

\section{Figures}




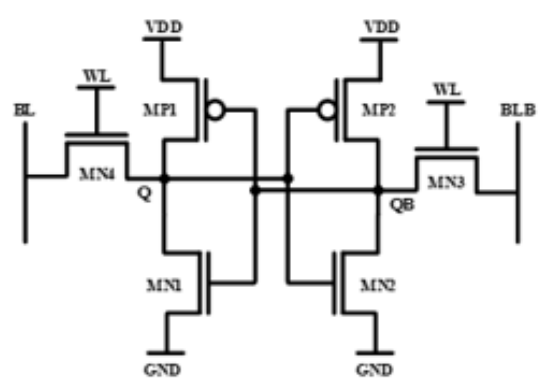

(a)

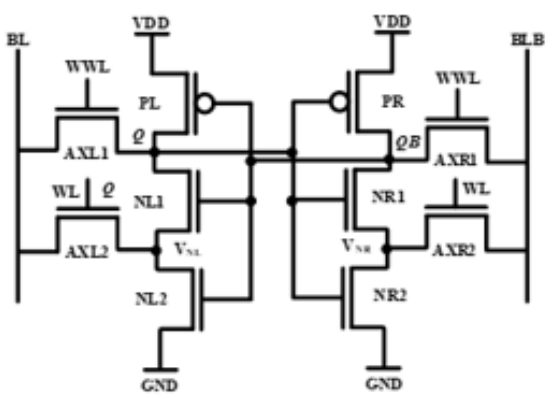

(c)

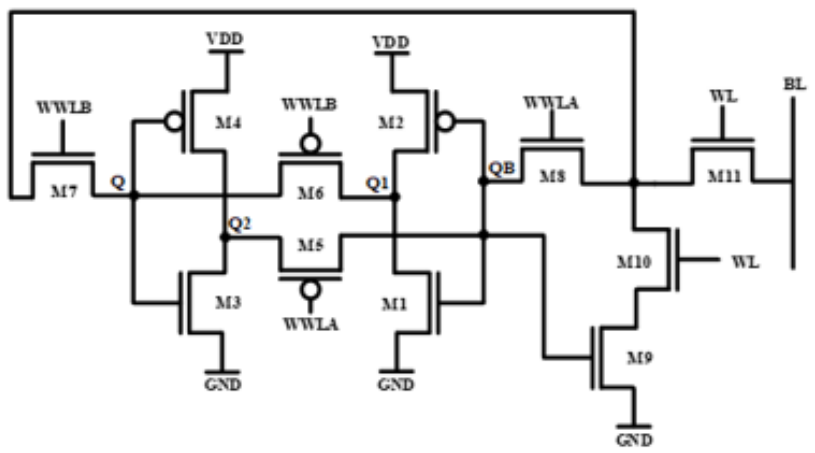

(e)

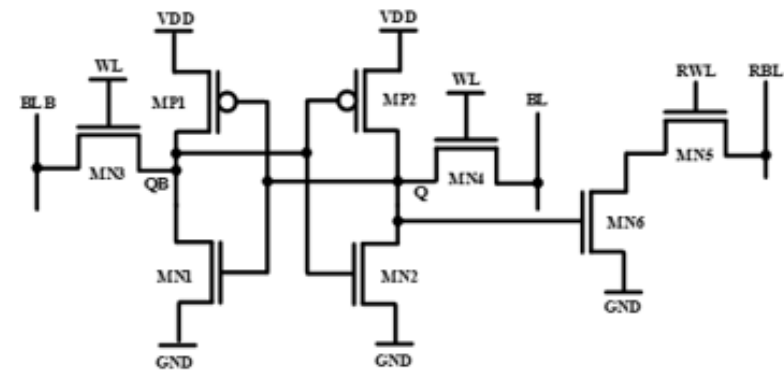

(b)

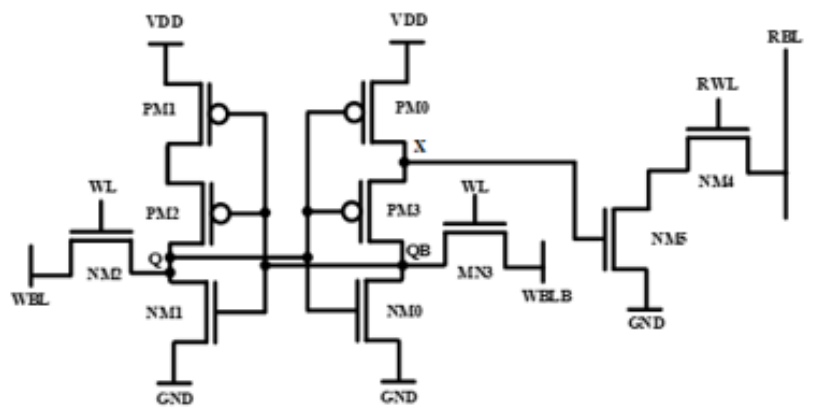

(d)

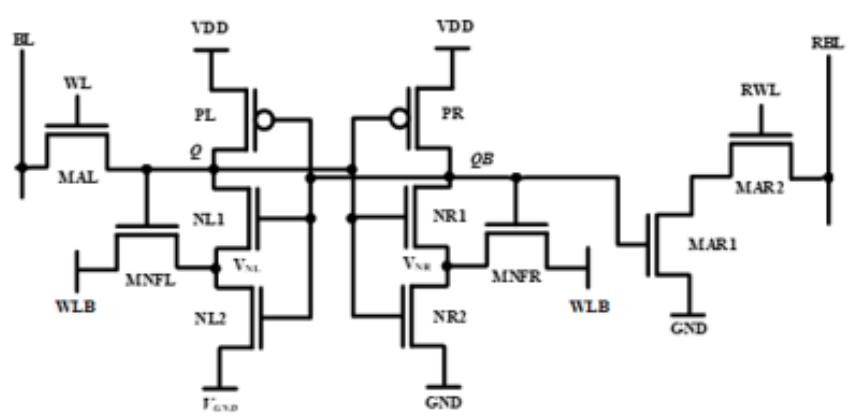

(f)

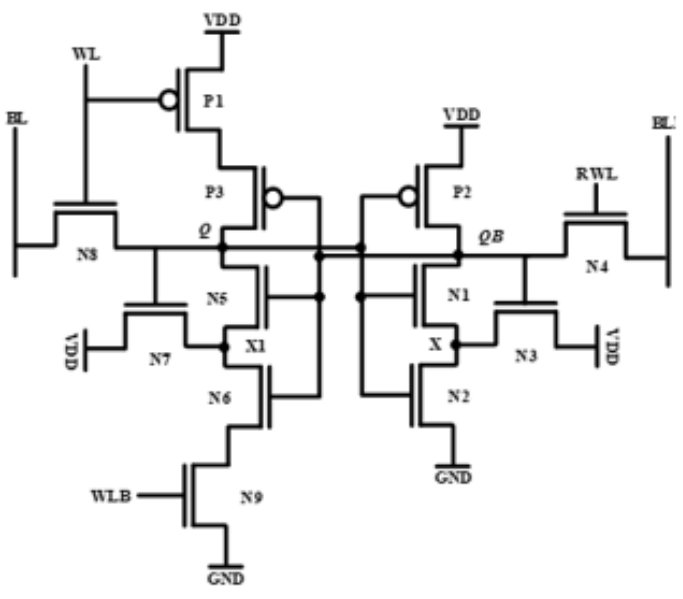

(g)

Figure 1

Schematic of the different SRAM cells. (a) 6T, (b) 8T, (c) ST2, (d) PPN10T, (e) FC11T, (f) ST11T, and (g) ST12T. 


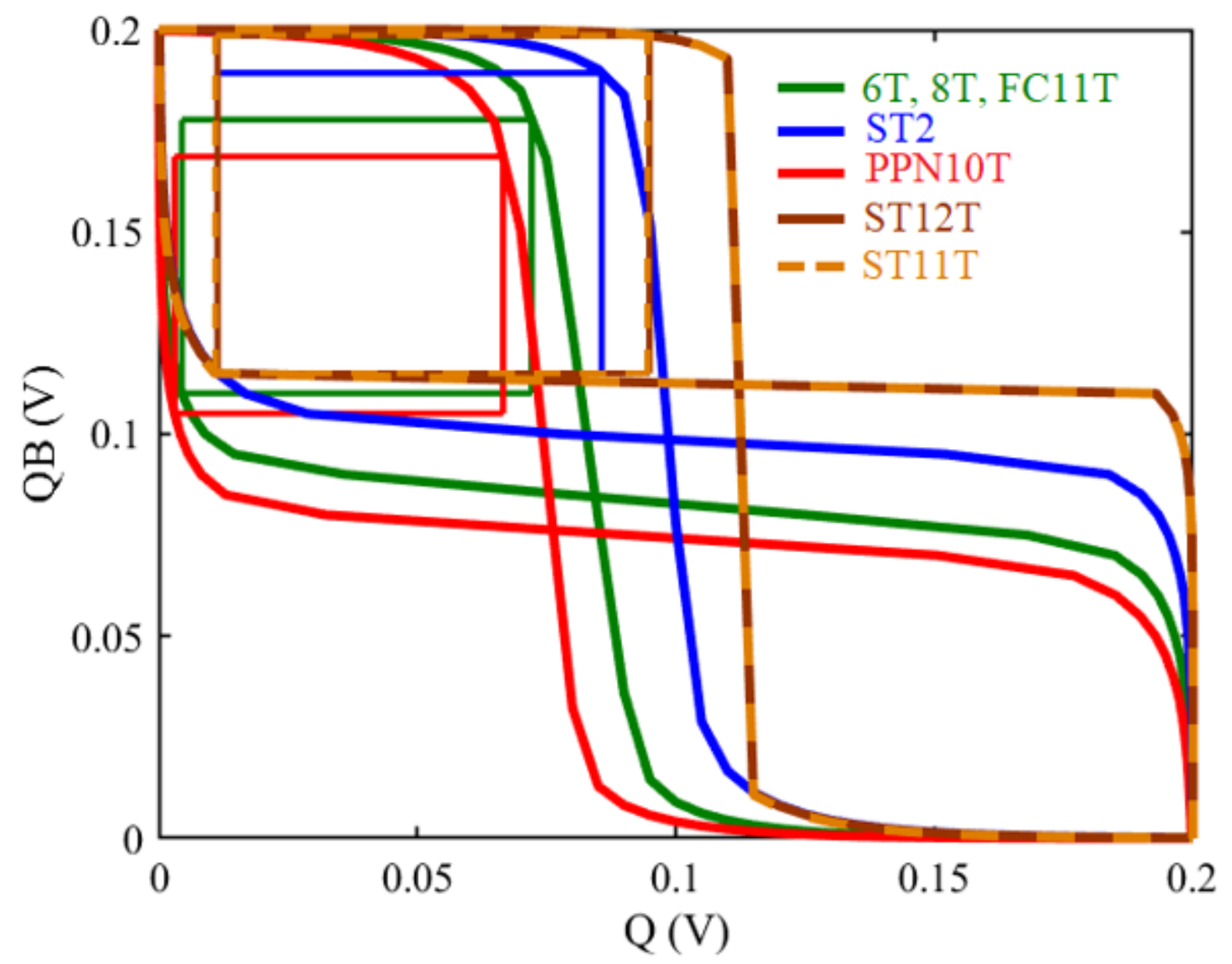

Figure 2

HSNM of the various SRAM cells at VDD $=0.2 \mathrm{~V}$. 


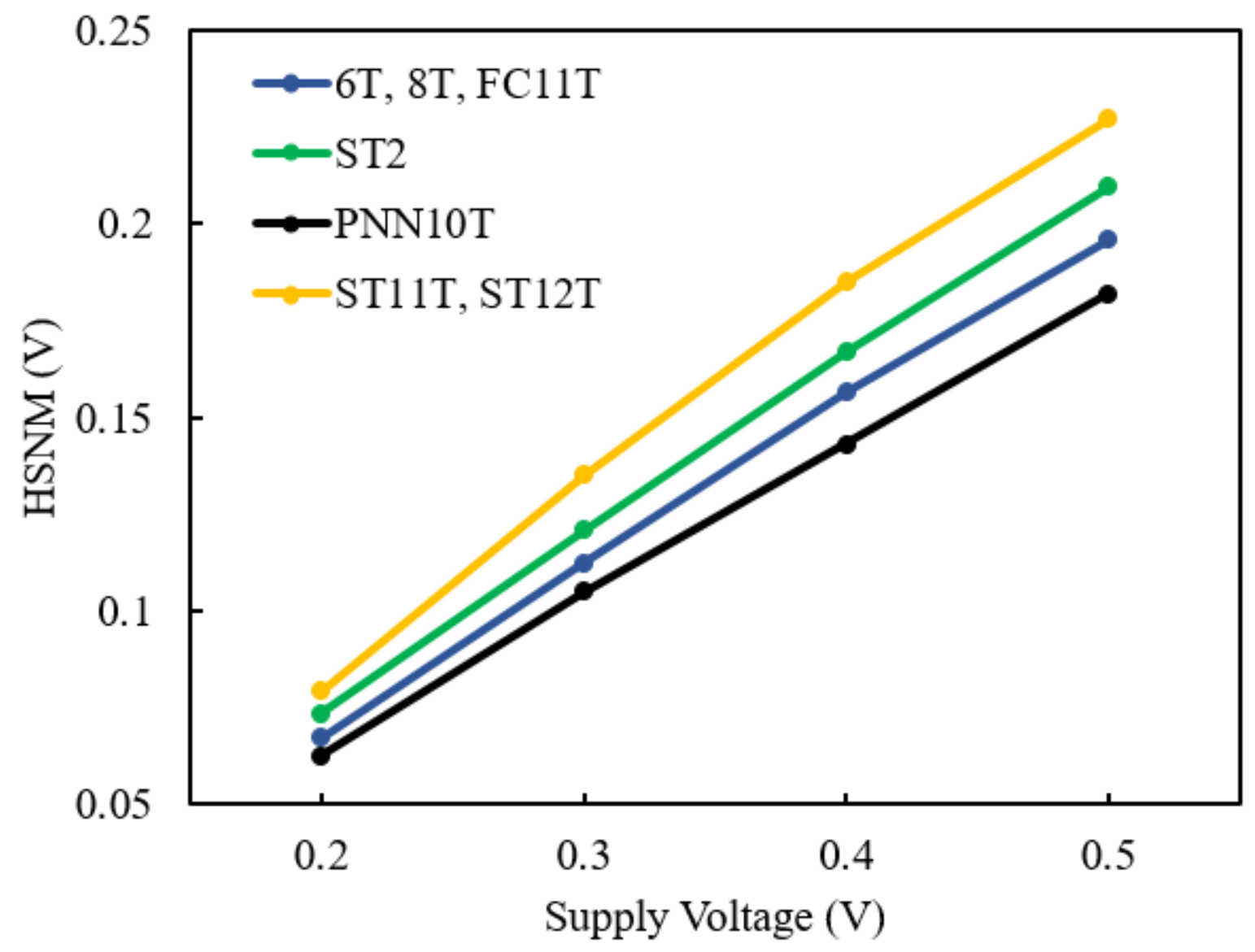

Figure 3

HSNM of the various SRAM cells at different VDD values. 


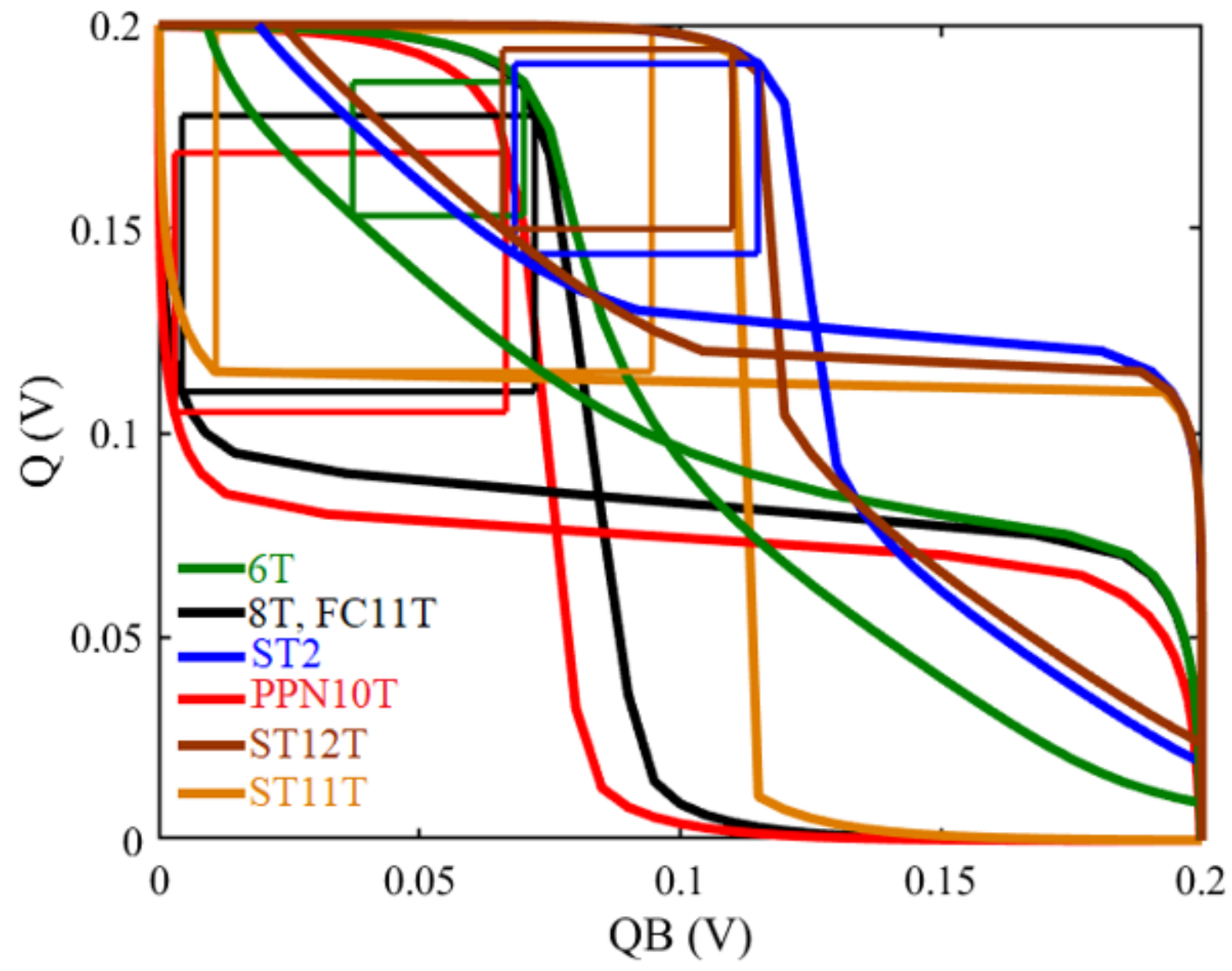

Figure 4

RSNM of the various SRAM cells at VDD $=0.2 \mathrm{~V}$. 


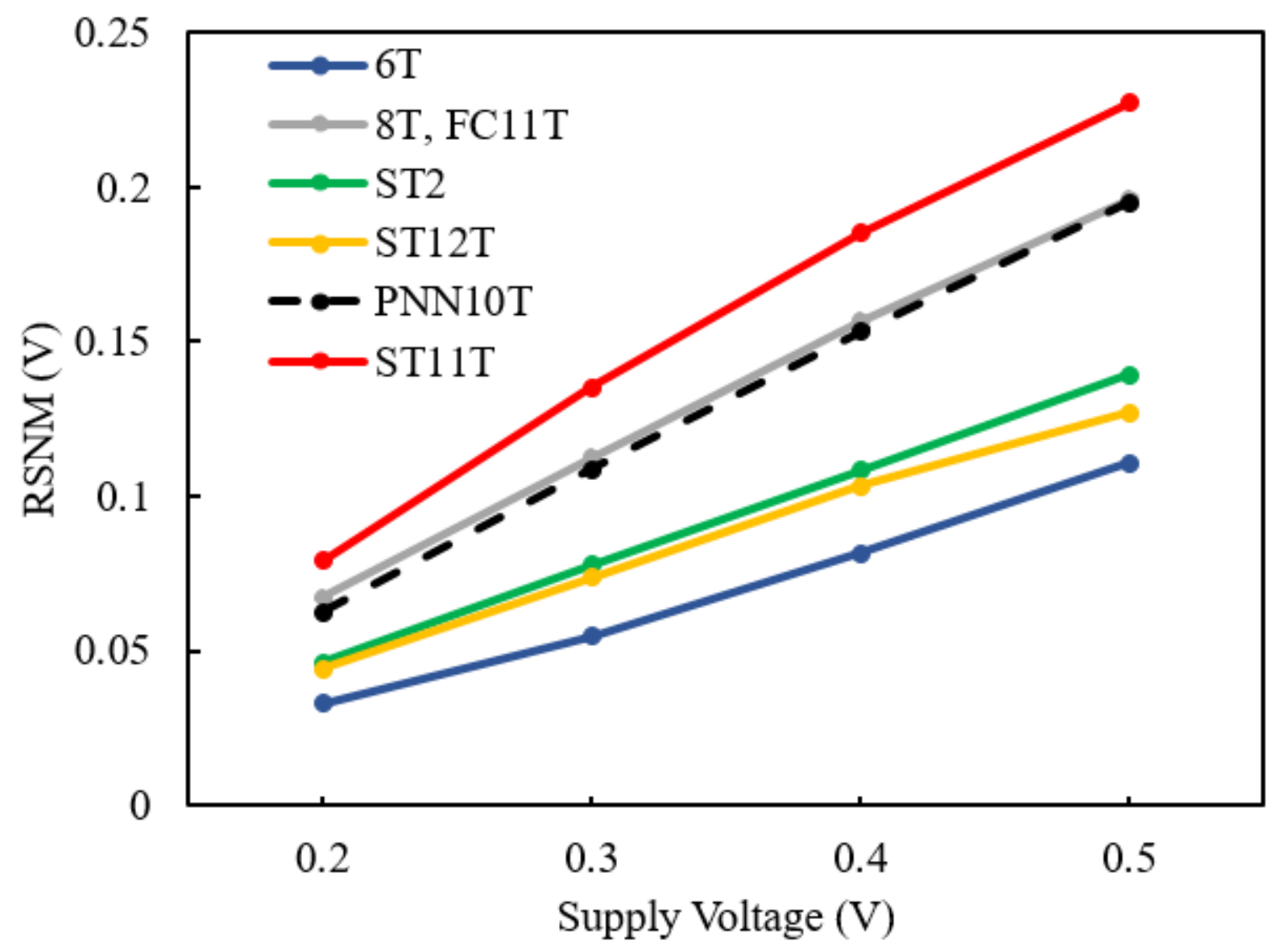

Figure 5

RSNM of the various SRAM cells at different VDD values. 


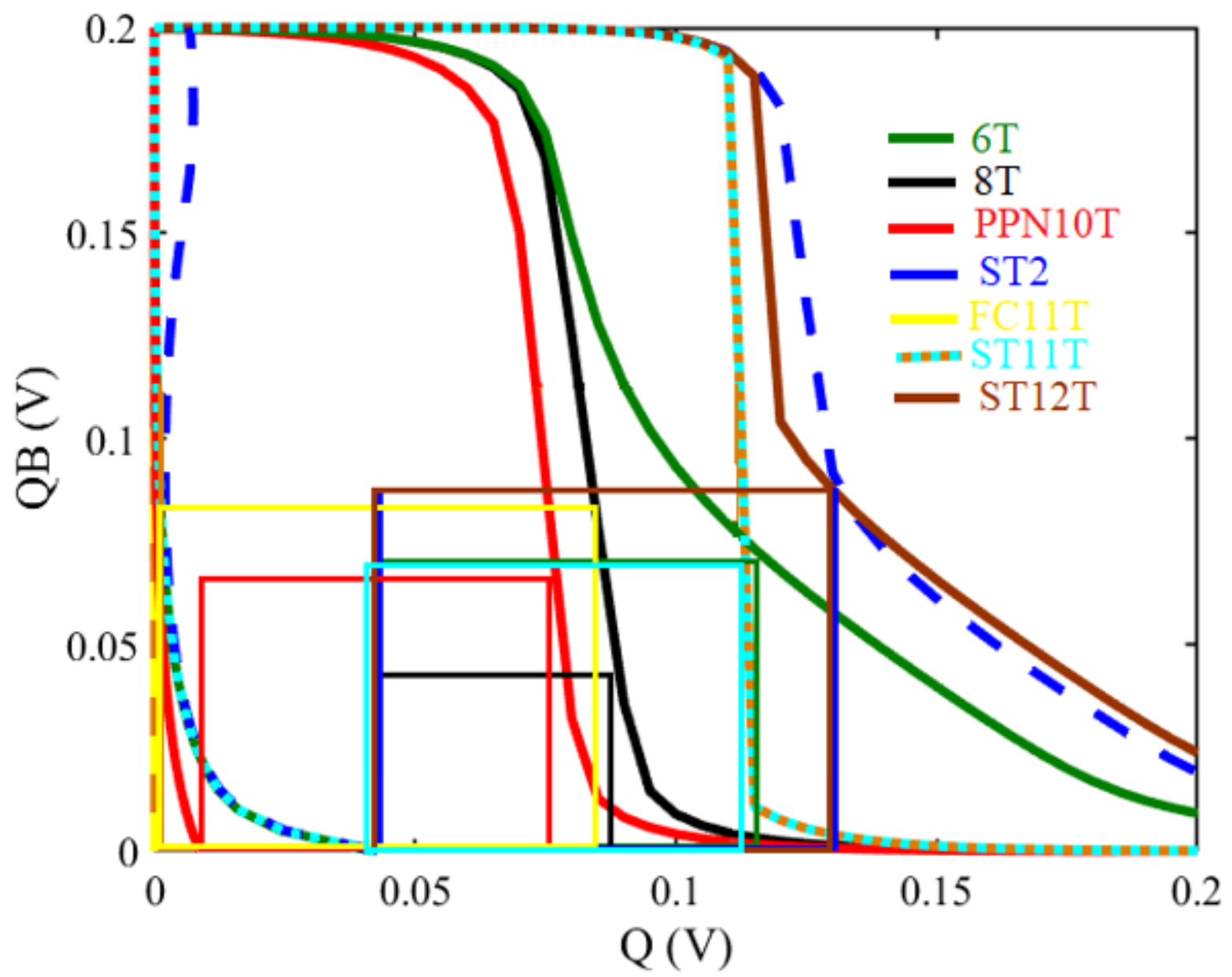

Figure 6

WSNM of the various SRAM cells at VDD $=0.2 \mathrm{~V}$. 


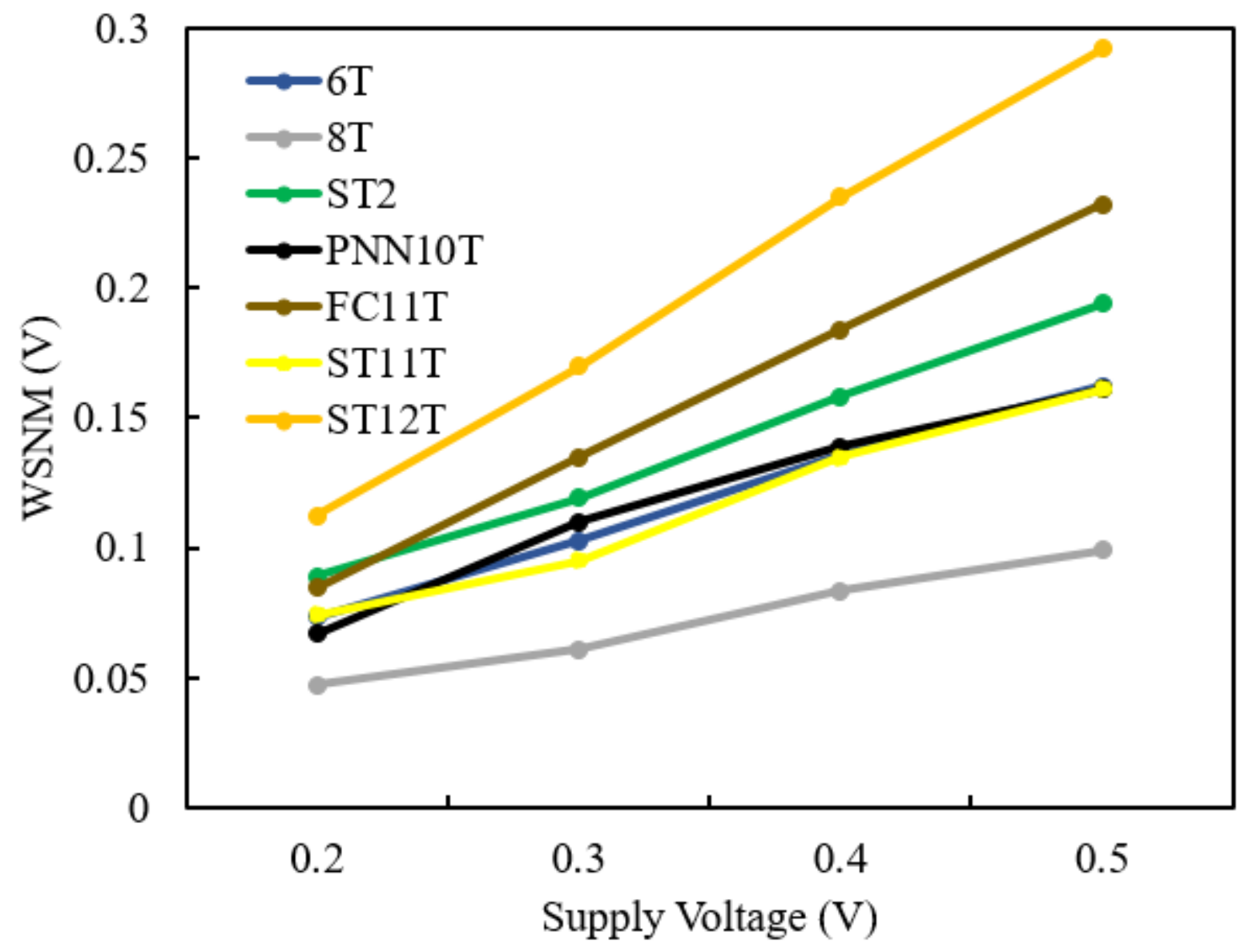

Figure 7

WSNM of the various SRAM cells at different VDD values. 


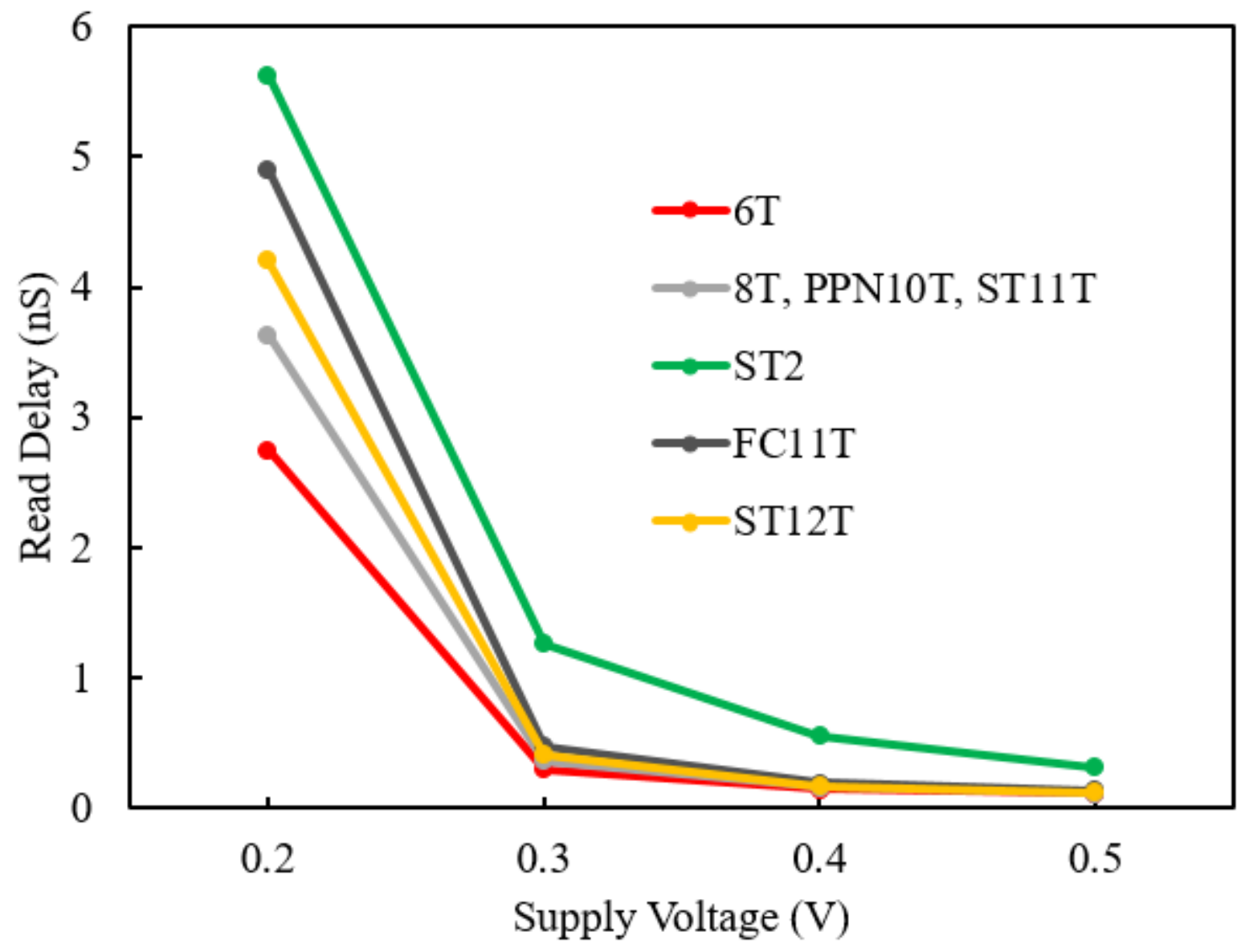

Figure 8

Read delay of the various SRAM cells at different VDD values. 


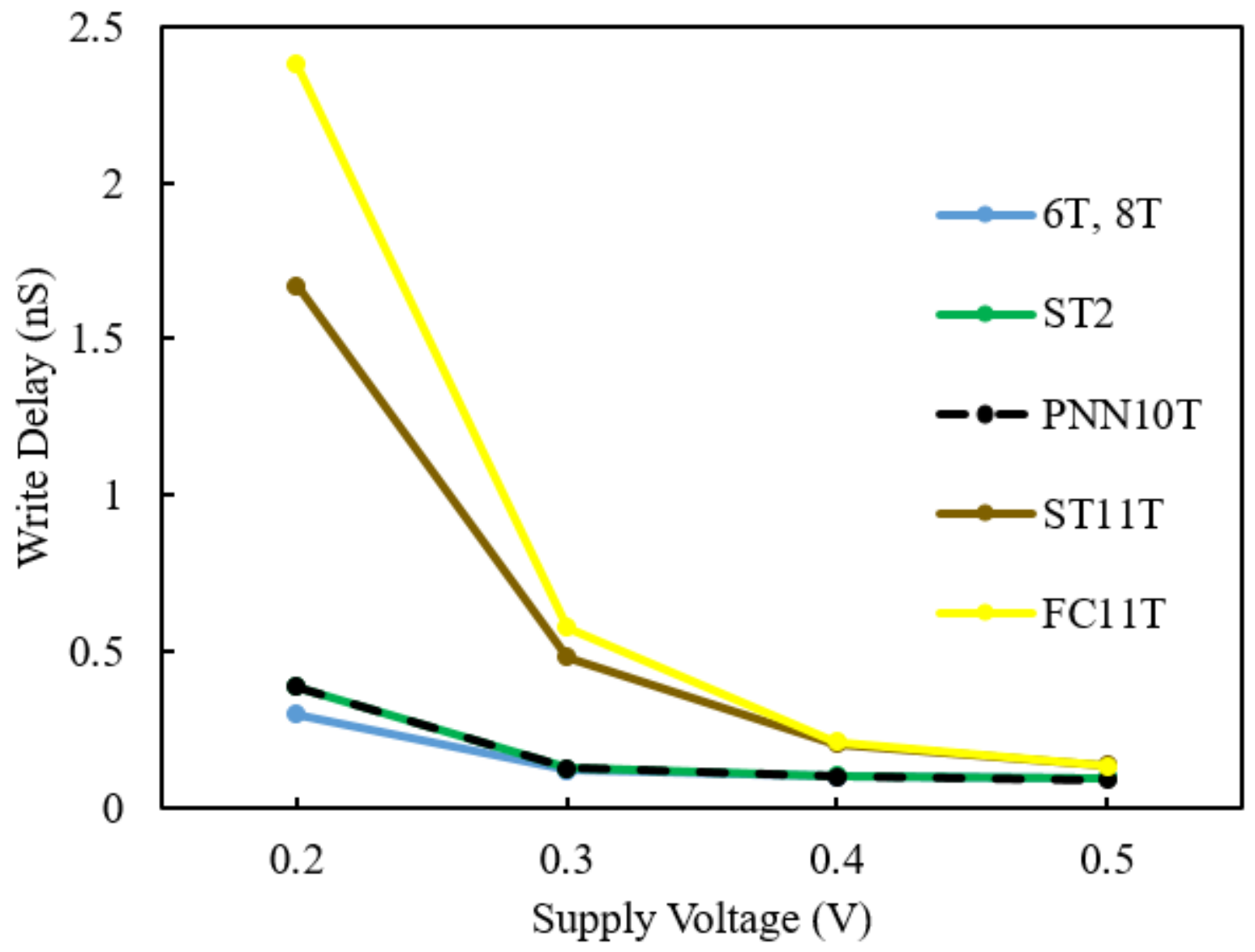

Figure 9

Write delay of the various SRAM cells at different VDD values. 


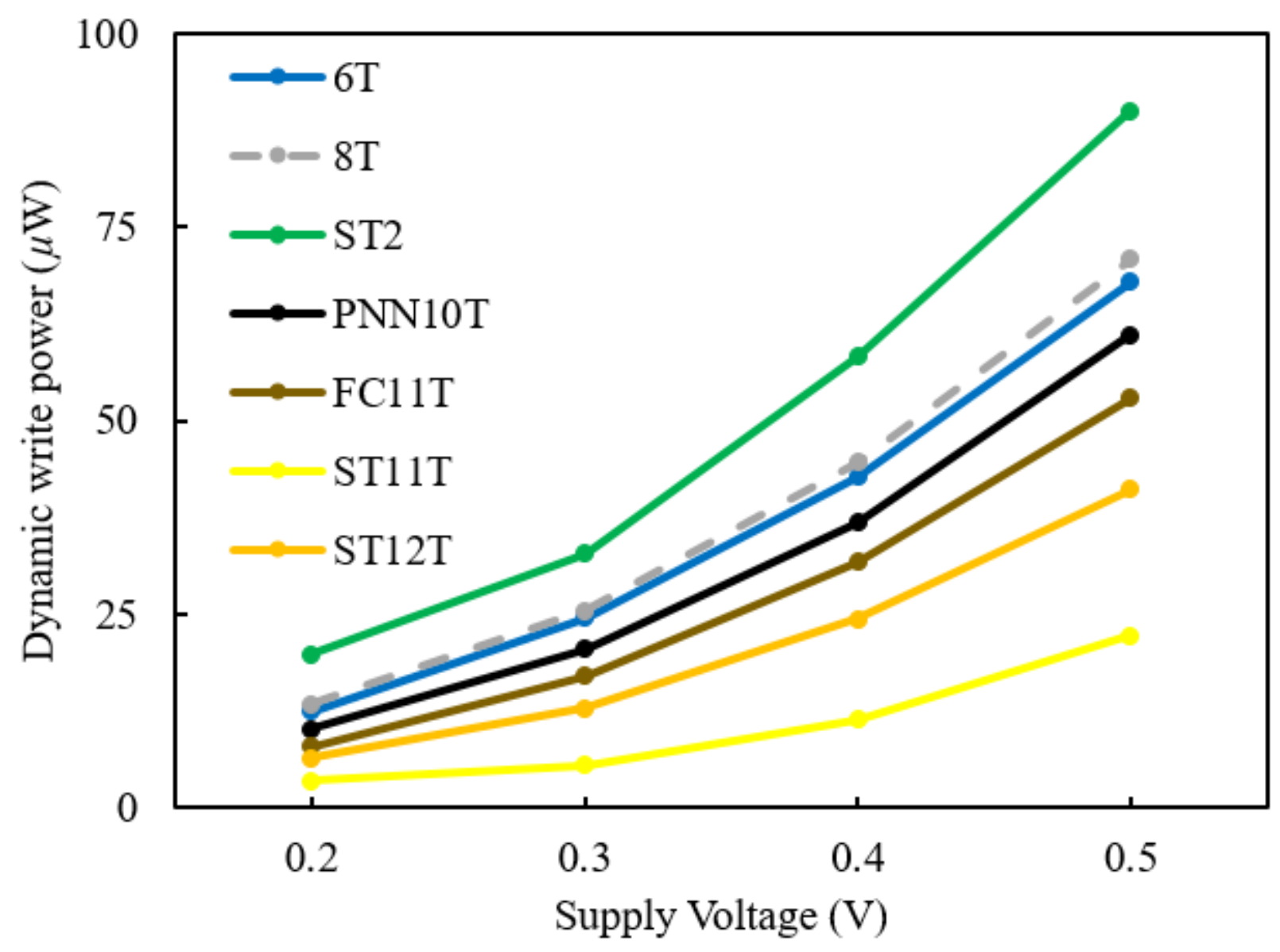

Figure 10

Dynamic write power of various SRAM cells at different VDD values. 


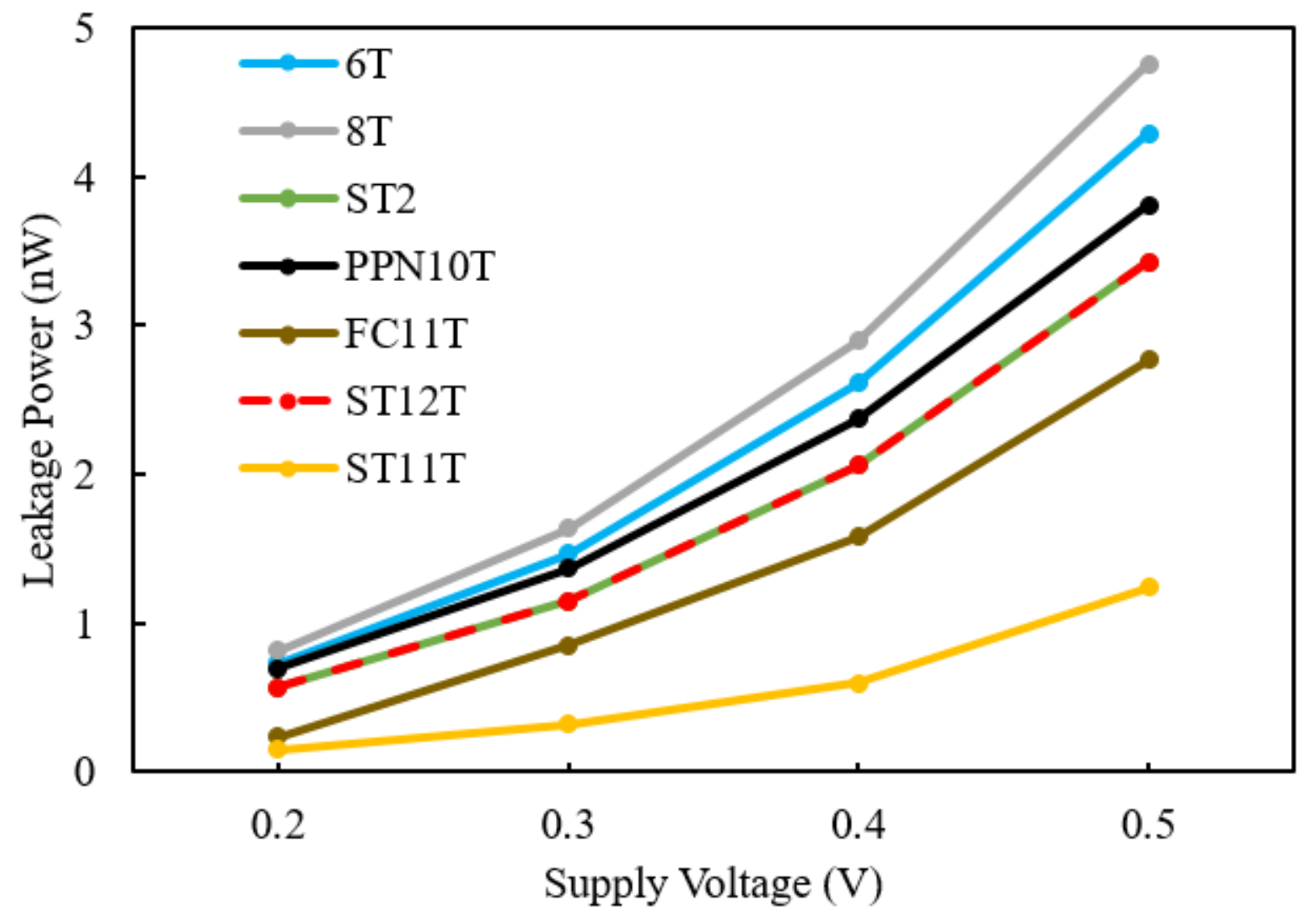

Figure 11

Leakage power of various SRAM cells at different VDD values. 


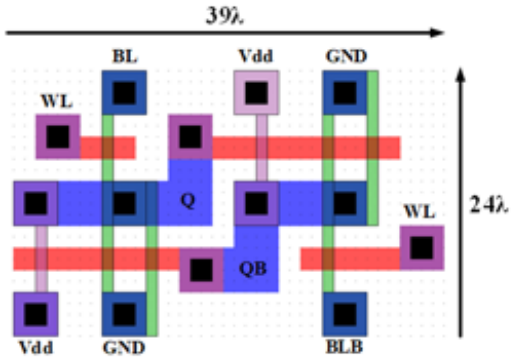

(a)

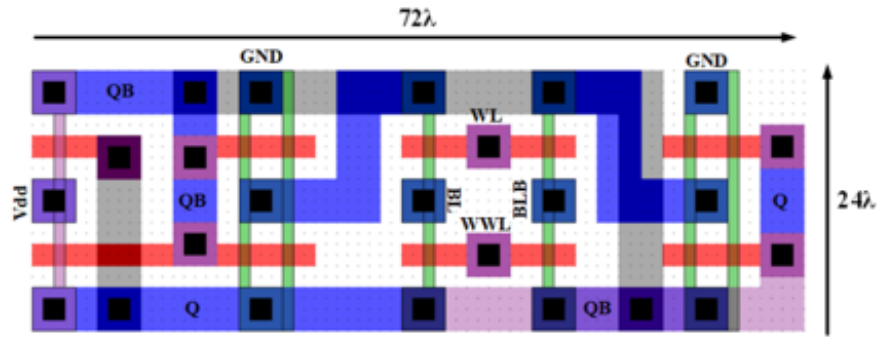

(c)

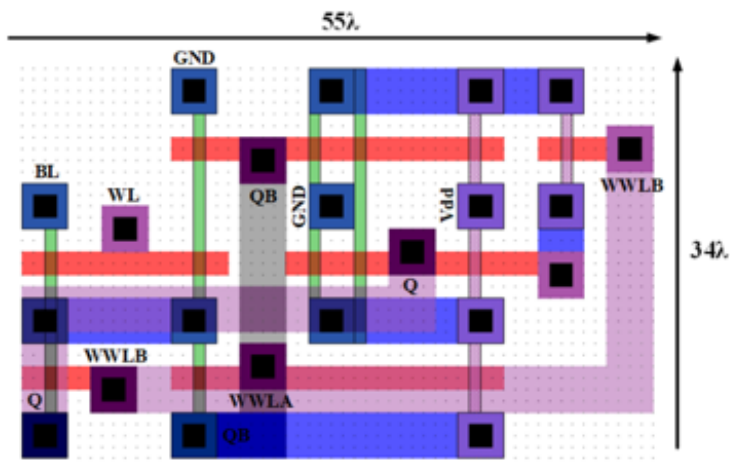

(e)

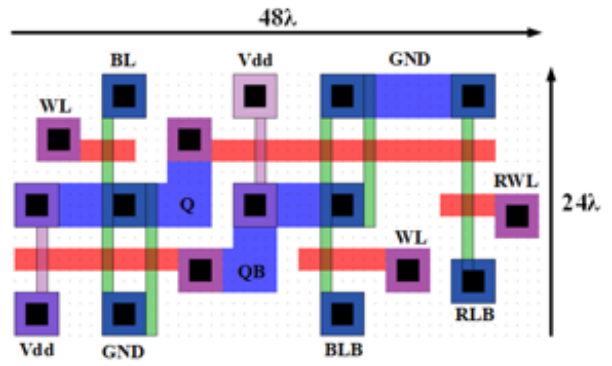

(b)

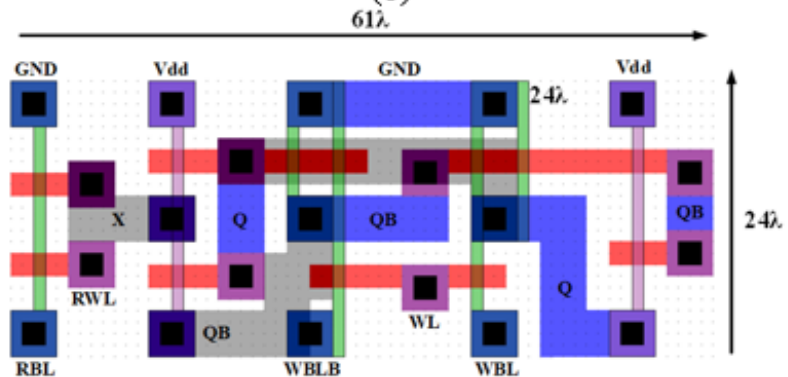

(d)

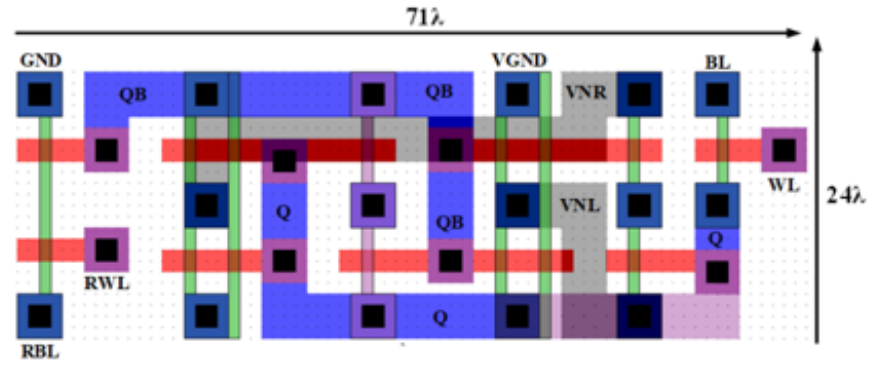

(f)

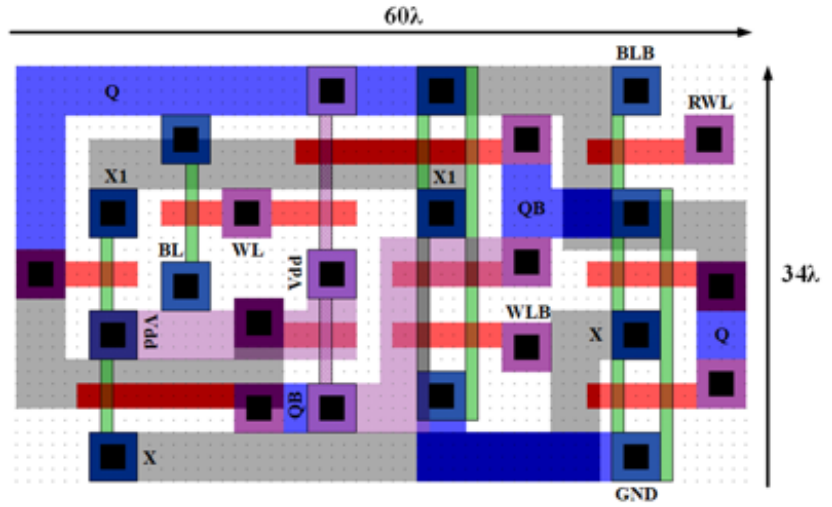

(g)

Figure 12

Layout of (a) 6T, (b) 8T, (c) ST2, (d) PPN10T, (e) FC11T, (f) ST11T, and (g) ST12T SRAM cells. 


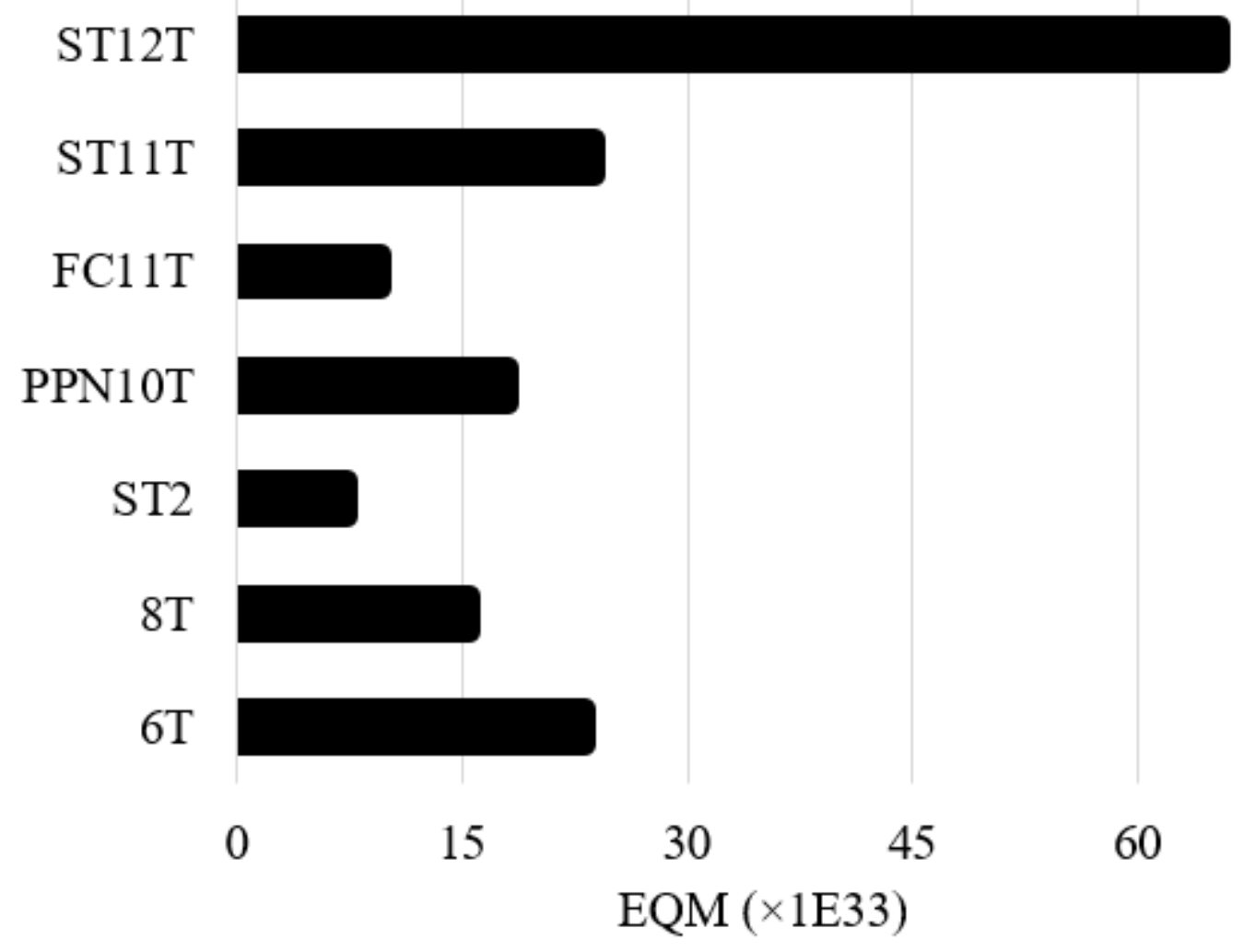

Figure 13

EQM for various SRAM cells at VDD $=0.2 \mathrm{~V}$. 